\title{
Fibronectin as an adjuvant in the diagnosis of oral inflammatory myofibroblastic tumor
}

\author{
Flávia-Caló-Aquino Xavier ${ }^{1}$, André-Caroli Rocha ${ }^{2}$, Norberto-Nobuo Sugaya ${ }^{2}$, Décio dos Santos-Pinto $\mathrm{Jr}^{3}$, \\ Suzana-Cantanhede-Orsini-Machado de Sousa ${ }^{3}$
}

${ }^{1}$ DDS, MsC, Department of Oral Pathology, School of Dentistry, University of São Paulo, Brazil

${ }^{2}$ DDS, PhD, Department of Stomatology, School of Dentistry, University of São Paulo, Brazil

${ }^{3}$ DDS, PhD, Department of Oral Pathology, School of Dentistry, University of São Paulo, Brazil

Correspondence:

Disciplina de Patologia Bucal

Faculdade de Odontologia da USP

Av. Prof. Lineu Prestes, 2227

São Paulo - SP, Brasil - 05508-900

f.calo@usp.br

Received: 13/02/2009

Accepted: 08/06/2009
Xavier FCA, Rocha AC, Sugaya NN, dos Santos-Pinto D, de Sousa SCOM. Fibronectin as an adjuvant in the diagnosis of oral inflammatory myofibroblastic tumor. Med Oral Patol Oral Cir Bucal. 2009 Dec 1;14 (12):e635-9.

http://www.medicinaoral.com/medoralfree01/v14i12/medoralv14i12p635.pdf

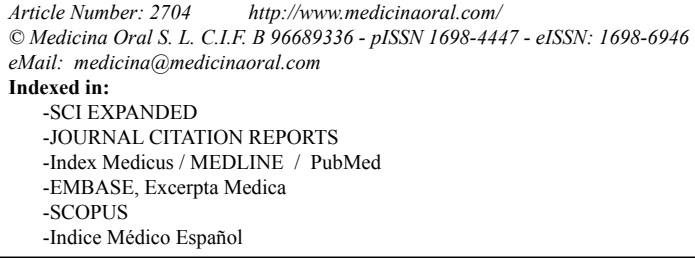

\begin{abstract}
Inflammatory myofibroblastic tumor is a distinctive lesion composed of myofibroblastic spindle shaped cells accompanied by inflammatory infiltrate that may arise in various organs. It is believed to be a noneoplastic inflammatory condition, although this is still controversial. The recognition of inflammatory myofibroblastic tumor as an entity is important especially to avoid unnecessary surgery. A few cases have been reported in the oral cavity. This report primarily presents a case of inflammatory myofibroblastic tumor that arose in the floor of mouth of a 23-year-old woman. The proliferating spindle cells were immunoreactive for vimentin, smooth muscle actin, and muscle specific actin and negative for desmin, AE1/AE3, S-100, CD68, MyoD1 and caldesmon. In an attempt to assess the potential neoplastic nature of this lesion, immunohistochemical expression of ALK protein was performed, although no immunoreactivity was detected. Also, the presence of well differentiated myofibroblasts identified by fibronectin is discussed, as well as the importance in establishing an immunoprofile to better consolidate the diagnosis. We conclude that the study of fibronectin in case series may aid the diagnosis as well as the prediction of the tumor course.
\end{abstract}

Key words: Inflammatory myofibroblastic tumor, myofibroblast, immunohistochemistry, fibronectin.

\section{Introduction}

Inflammatory myofibroblastic tumor (IMT) is a rare entity included in a heterogeneous group of spindle cell proliferations, which encompasses a broad phenotypic and biologic spectrum of diseases ranging from reactive lesions to benign neoplasms (1). Tumours and tumourlike lesions of myofibroblasts may present diagnostic difficulty because of their rarity and uncertainties in identifying the myofibroblast (2). So, immunohistochemistry and electron microscopy are emphasized as being important for maximum diagnostic confidence in some myofibroblastic lesions (2). The expression of myofibroblast by characteristic proteins as $\alpha$-smoothmuscle actin and fibronectin are essential to diagnosing myofibroblastic lesions $(3,4)$. The purpose of this study is primarily to present a case of IMT in the floor 
of the mouth, describing its clinical aspect, immunophenotype and management, showing for the first time myofibroblastic fibronectin expression contributing to the knowledge and characterization of this entity.

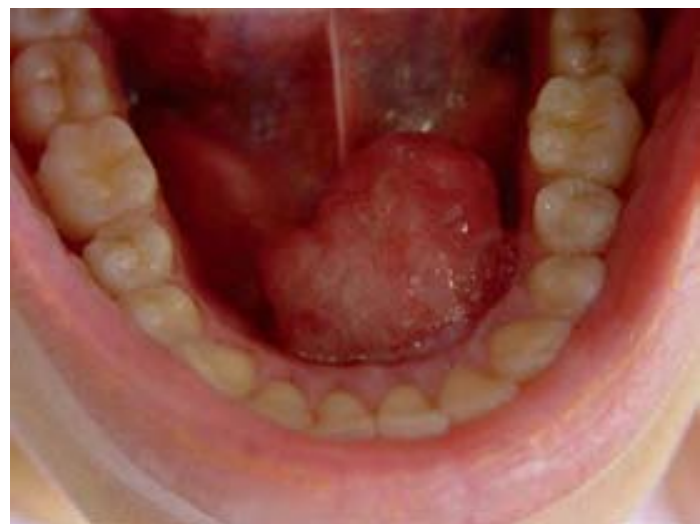

Fig. 1. Clinical view of the mandible showing the round pedunculated reddish mass with a rubbery consistency, attached to the floor of mouth mucosa.

\section{Case report}

A 23-year-old white woman presented for evaluation of a painless swelling with a three-week history of progressive growing on the floor of the mouth. Her medical and surgical histories were unremarkable, and she denied any previous lesions or local trauma. Intraoral examination revealed a $3 \mathrm{~cm}$ pedunculated reddish exophytic mass, with a rubbery consistency, attached to the mucosa (Fig. 1). The overlying mucosa presented ulcerated areas with no discharge.

On the basis of the clinical findings, glandular neoplasm, noneoplastic proliferative process or malignant tumor were considered as differential diagnosis. An incisional biopsy was performed and, microscopic examination revealed an ulcerated nodular mass composed of proliferating spindle cells in a predominantly myxoid stroma admixed with a variable amount of acute and chronic inflammatory cells along with a rich vascular network (Fig. 2A). Towards the periphery, the lesion was more cellular with a focal fascicular arrangement of the spindle cells. In all fields, an inflammatory component was prominent and was formed mainly by neutrophils and lymphocytes with a few plasma cells, histiocytes and

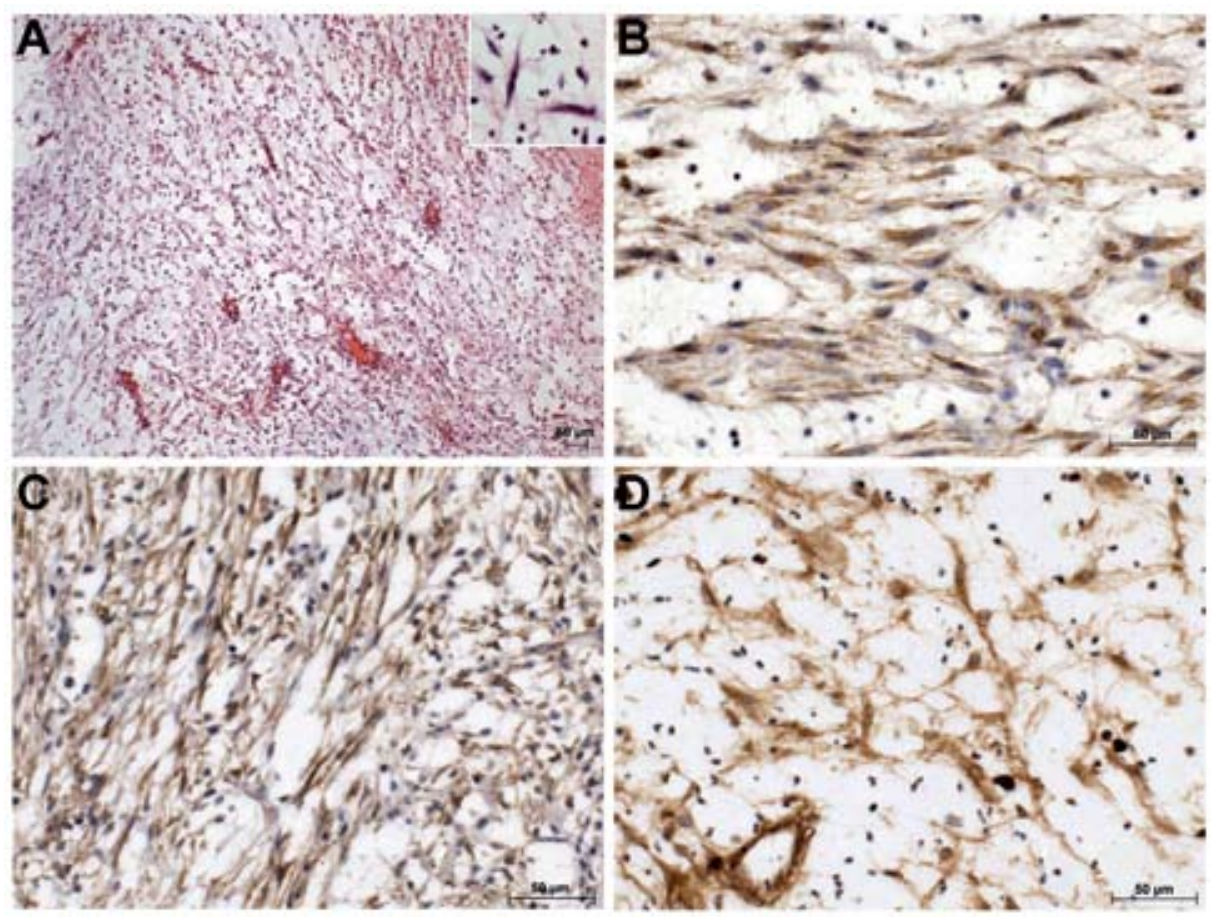

Fig. 2. Photomicrographs of Inflammatory myofibroblastic tumor. A, spindle tumor cells scattered in a loosely and myxoid background with inflammatory proliferation; in detail, myofibroblasts and eosinophils (hematoxylin and eosin stain). B, immunohistochemical staining of SMA in spindled myofibroblasts. C, diffuse immunostaining of HHF-35 also showed in spindled myofibroblasts. D, strong immunohistochemical staining of Fibronectin in spindled myofibroblasts. Streptavidin-biotin (B, C and D). Scale bars indicate $50 \mu \mathrm{m}$. 


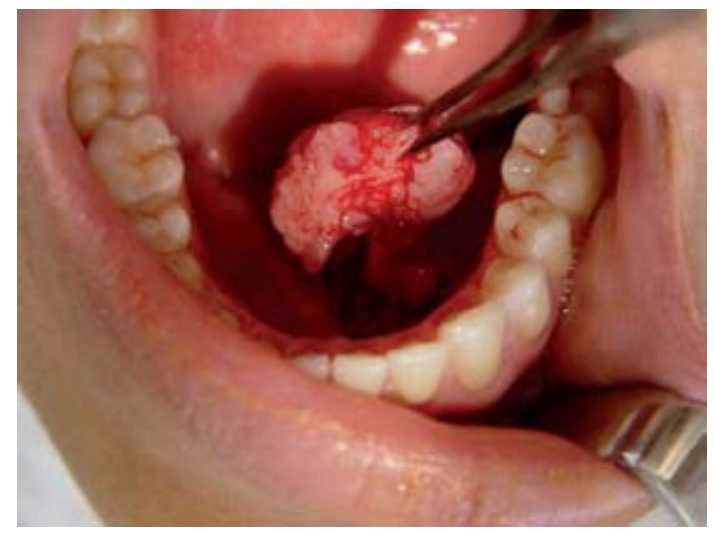

Fig. 3. Trans-operative aspect of the inflammatory myofibroblastic tumor during surgical excision.

eosinophils. The spindle cells exhibited plump tapering vesicular nuclei and were haphazardly arranged (Fig. 2A). Non-cohesive larger stellate or polygonal cells with ovoid vesicular nuclei and prominent nucleoli were scattered. Nuclear atypia and mitosis were not evident.

Additionally, immunohistochemistry was performed with proliferating spindle cells showing intense and diffuse positivity for vimentin (M0725, DakoCytomation, Carpinteria, CA, USA), smooth muscle actin (SMA; M0851, DakoCytomation) (Fig. 2B) and muscle specific actin (HHF-35; M0635, DakoCytomation) (Fig. 2C). The immunostaining for desmin, however, was negative as were also negative reactions to AE1/AE3 (M3515, DakoCytomation), S-100 (Z311, DakoCytomation), CD68 (M0814, DakoCytomation), MyoD1 (M3512, DakoCytomation), and caldesmon (M3557, DakoCytomation). In an attempt to delineate the potential neoplastic nature of this lesion, we assessed the immunohistochemical expression of ALK protein (M7195, DakoCytomation), although none reactivity was found. A diagnosis of IMT was established. Furthermore, fibronectin (A0245, DakoCytomation) immunostaining was performed, showing strong positivity in spindle cells (Fig. 2D), corroborating with a myofibroblastic phenotype.

A surgical excision was carried out (Fig. 3) and postoperative histopathological examination confirmed the initial diagnosis of IMT. The postoperative course was unremarkable. The patient was satisfied with her quality of life and remained disease free at the time of her last follow-up, 2 years after surgery.

\section{Discussion}

Oral IMT is a very rare lesion which along with its nonspecific clinical appearance may pose difficulties to diagnosis and management. Its rapid growth rate may simulate a malignant disorder and therefore warrants a comprehensive histopathologic assessment (5). Originally reported in the lung, extra-pulmonary IMTs have been described (1), including head and neck region (6), mainly in the aerodigestive tract, major salivary glands and soft tissues of the neck $(1,7,8)$. A limited number of patients with oral IMT have been reported (5), thus contributing with the unspecified pattern of clinical aspects and clues to diagnosis. In the oral mucosa, the most reported sites of occurrence are submandibular region (9), parotid duct (7), retromolar area (10), alveolar mucosa of the molar region (5), tongue (11), maxilla (7) and the hard palate (6). Typically, oral IMTs have been described as a well circumscribed, solitary nodule or mass, frequently pedunculated, and rubbery consistency, as the present one, have been reported (5).

The etiology of most oral IMTs remains controversial. Some authors widely consider it a benign neoplastic lesion (6), and others support a reactive etiology (10), since the rapid growth and subsequent indolent behavior of most oral IMT are similar to that of many common reactive oral lesions (5). Differently, in other sites, this condition can behave in a more aggressive or even malignant way (11), constituting a true neoplastic process due to the potential for local recurrence, development of multifocal tumors, infiltrative local growth, and even distant metastasis (12).

Histologically, various aspects can be seen. In the current case one of the patterns was observed; loosely arranged myofibroblasts in an edematous and myxoid background with plasma cells, lymphocytes, eosinophils, and blood vessels $(5,10)$. A second configuration that can occur is distinguished by the presence of dense aggregates of spindle cells arranged in a variable myxoid or collagenized background, admixed with a distinctive inflammatory infiltrate (13). Finally, a third pattern of IMT is characterized by prominent collagen sheets, resembling scar tissue, with scattered plasma cells and eosinophils (5). Cytological atypia with nuclear pleomorphism and increased mitotic activity are uncommon features $(5,6)$, and may be associated with malignant transformation (5).

Immunoprofile is helpful in the establishment of the diagnosis of IMT especially by the identification of myofibroblasts. The myofibroblast is becoming recognized as a target for translational medicine, since it appears as a significant cellular participant in granulation tissue and several other human diseases (14). Previously, the characterization of the fully differentiated myofibroblast immunophenotype included positivity to vimentin and $\alpha$-smooth-muscle actin, and negativity to smoothmuscle myosin and usually desmin (3). However, a spindle cell positive to SMA is an imprecise definition for the myofibroblast since a number of normal cells also express it (14). Additionally, lesions first regarded as myofibroblastic are shown to vary in their level of differentiation, and some appear to be smooth-muscle rather than myofibroblastic (2). Desmin negativity rather than positivity should be seen as a more appropriate 
indicator of myofibroblastic differentiation (14). Finally, as recently suggested, the main characteristics of the myofibroblast include a spindled or stellate morphology, immunostaining for alpha-smooth-muscle actin and the extra domain A variant of cellular fibronectin, and an ultrastructure of rough endoplasmic reticulum, peripheral contractile filaments and the cell-to-matrix junction known as the fibronexus, being important for maximum diagnostic confidence in some myofibroblastic lesions (2).

The immunophenotype of the IMT presented herein is in accordance with the identification of a myofibroblast $(2,14)$, and also by other IMT reports $(5,13)$ with strong diffuse cytoplasmic reactivity to vimentin, SMA and HHF-35 in a focal or diffuse pattern and also negativity to desmin, although the latest could be identified in many cases, as well as focal cytokeratin immunoreactivity (15). Myogenin, myoglobin, and S-100 protein are also negative $(1,15)$, as were in the present case. Futhermore, we believe that more investigations on tumor behavior should take into account the fibronectin immunoprofile, since it is known that this protein shows properties that could precipitate a cascade leading to a number of cellular activities, such as differentiation, migration, mitosis all important to tumor progression (14). Recently, this marker has been associated to the invasive phenotype of oral carcinoma cell lines, although the authors could not establish if the presence of its expression is an associated phenomena or a causative agent in the invasive process (4).

Immunohistochemical cytoplasmic positivity for ALK (Anaplastic lymphoma kinase) is linked to neoplastic transformation since it provides strong evidence for an oncogenic activating mechanism (5). Thus, at least a proportion of IMTs presenting ALK genetic alterations are genuine neoplasms (15). However, ALK expression has been detected in approximately $50 \%$ of IMTs, and therefore shows not to be a specific marker for this lesion $(1,15)$. The present case did not demonstrate immunoreactivity for ALK, as shown by most of the oral cases reported in the literature with only a few reports of oral IMT ALK-positive $(5,13)$. Whether ALK-positive IMTs represent distinct clinicopathologic entities, with different therapeutic and prognostic implications, warrants further investigation (13) in order to clarify the contribution of ALK deregulation to the pathogenesis of oral IMT (5).

In addition to the controversy regarding the biologic nature of IMT, proliferating spindle cells may mimic a sarcoma, potentially leading to a diagnostic dilemma (13). Various benign and malignant spindle cell proliferations should be included in the differential histological diagnosis scheme of oral IMT such as proliferative fasciitis, nodular fasciitis, infantile fibromatosis, myofibromatosis, Rosai-Dorfman disease, fibrous histiocy- toma, solitary fibrous tumor, follicular dendritic cell tumor, low-grade myofibroblastic sarcoma, fibrosarcoma, leiomyosarcoma, rhabdomyosarcoma, and spindle cell carcinoma $(4,10)$. So, the knowledge of the different histologic patterns of IMT, identification of a predominant inflammatory component and immunohistochemical study allow distinction between these entities, being helpful to the accurate diagnosis (5), as emphasized by the present report, and also preventing a more aggressive surgical procedure.

Management of IMT should entail complete surgical resection $(5,6)$, and since IMT may present a low-grade malignancy, longer follow-up is needed (11). Extrapulmonary IMTs seem to exhibit a more favorable clinical course and treatment outcome, evolving with lower rates of recurrence, malignant transformation, metastasis, and mortality (5). One noteworthy feature is that oral IMT seems to behave differently from IMT occurring elsewhere in body (11), displaying variable local recurrence and not reported metastasis (6). There may be a need to recognize it as a separate entity. The diversity of IMTs microscopic appearance and the lack of correlation between these variables and the clinical evolution of these tumors to date, encourage further research on biomarkers, such as fibronectin, that might provide guidelines to tumor progression, treatment and prognosis.

\section{References}

1. Coffin CM, Humphrey PA, Dehner LP. Extrapulmonary inflammatory myofibroblastic tumor: a clinical and pathological survey. Semin Diagn Pathol. 1998;15:85-101.

2. Eyden B, Banerjee SS, Shenjere P, Fisher C. The myofibroblast and its tumours. J Clin Pathol. 2009;62:236-49.

3 . Eyden B. The myofibroblast: phenotypic characterization as a prerequisite to understanding its functions in translational medicine. $\mathrm{J}$ Cell Mol Med. 2008;12:22-37.

4. Brooks JK, Nikitakis NG, Frankel BF, Papadimitriou JC, Sauk JJ. Oral inflammatory myofibroblastic tumor demonstrating ALK, p53, MDM2, CDK4, pRb, and Ki-67 immunoreactivity in an elderly patient. Oral Surg Oral Med Oral Pathol Oral Radiol Endod. 2005;99:716-26.

5. Cable BB, Leonard D, Fielding CG, Hommer DH. Pathology forum: quiz case 1. Diagnosis: inflammatory myofibroblastic tumor (IMT). Arch Otolaryngol Head Neck Surg. 2000;126:900, 904-5.

6. Shek AW, Wu PC, Samman N. Inflammatory pseudotumour of the mouth and maxilla. J Clin Pathol. 1996;49:164-7.

7. Barrios-Sánchez GM, Dean-Ferrer A, Alamillos-Granados FJ, Ruiz-Masera JJ, Zafra-Camacho FM, García de Marcos JA, et al. Inflammatory pseudotumor of the parotid gland. Med Oral Patol Oral Cir Bucal. 2005;10:371-5.

8. Earl PD, Lowry JC, Sloan P. Intraoral inflammatory pseudotumor. Oral Surg Oral Med Oral Pathol. 1993;76:279-83.

9. Ide F, Shimoyama T, Horie N. Inflammatory pseudotumor in the mandibular retromolar region. J Oral Pathol Med. 1998;27:508-10.

10. Pankaj C, Uma C. How to manage oral inflammatory myofibroblastic tumor (inflammatory pseudotumor)?. Oral Dis. 2001;7:315-6. 11. Frigui M, Khabir A, Jallouli M, Mnif Z, Hdiji S, Elloumi M, et al. [Recurrent inflammatory myofibroblastic tumor with renal, retroperitoneal and lymph node involvement]. Rev Med Interne. 2009;30:372-6. 
12. Poh CF, Priddy RW, Dahlman DM. Intramandibular inflammatory myofibroblastic tumor--a true neoplasm or reactive lesion?. Oral Surg Oral Med Oral Pathol Oral Radiol Endod. 2005;100:460-6.

13. Skalli O, Schürch W, Seemayer T, Lagacé R, Montandon D, Pittet $\mathrm{B}$, et al. Myofibroblasts from diverse pathologic settings are heterogeneous in their content of actin isoforms and intermediate filament proteins. Lab Invest. 1989;60:275-85.

14. Chan JK, Cheuk W, Shimizu M. Anaplastic lymphoma kinase expression in inflammatory pseudotumors. Am J Surg Pathol. 2001;25:761-8.

15. Nielsen JD, Moeslund M, Wandall HH, Dabelsteen S. Influences of tumor stroma on the malignant phenotype. J Oral Pathol Med. 2008;37:412-6 